\title{
Operating Nature of Meaning
}

\author{
ZHANG Zhi-xiu \\ Southwest University, Chongqing, China
}

\begin{abstract}
Meaning should depend on speakers to operate its interactional and communicational function; speakers usually depend on intended meaning, but not linguistic meaning or literal meaning to engender the meaning and the form of a linguistic expression. Meaning can emerge from image schema, emotion, thought, or instant structures, so meaning generally possesses four kinds of emerging resources. Emotion, thought, image schema, and instant structure are not only the meaning emerging resources, but also the parts of meaning of a linguistic expression.
\end{abstract}

Keywords: operating nature, meaning, emerging resource

\section{Introduction}

Meaning can be instantiated as linguistic meaning or literal meaning to involve the activities of interaction and communication; but, people in fact depend on intended meaning to take part in the interaction and communication in daily time. Intended meaning can be conveyed partly with linguistic expressions, partly with nonlinguistic expressions. When speakers are in the interactional and communicational process, they usually use intended meaning, not linguistic meaning or literal meaning to engender the meaning and the form of linguistic expressions, because meaning possesses four kinds of emerging resources, which are image schema, emotion, thought, or instant structures.

\section{Linguistic Meaning or Intended Meaning}

According to Johnson (1987), linguistic meaning in the broadest sense includes all structures we catch, these structures endow us with understanding, bestow our experience definiteness, and connect different experience domain. On this account, these structures establish sequential measure and the unity for us to understand the world; also they can endow our experience orders. However, Johnson points out that linguistic meaning in a more broadly sense, is just one case in meanings, which means that some meanings cannot be embedded in linguistic organizers or instantiated as linguistic meaning. Linguistic meaning is short for interaction and communication, even though intended meaning can be conventionalized as linguistic meaning, and linguistic meaning can be evoked by intended meaning. So there are some other kinds of meaning for better interaction and communication, which may be intended meaning, I think. That is, the meaning in the interactional and communicational process factually is intended meaning, but not linguistic meaning. Because intended meaning can convey the meaning that linguistic meaning cannot convey, which means intended meaning sometimes can be seen as the sum of linguistic meaning plus the meaning that linguistic organizers cannot conventionalized.

ZHANG Zhi-xiu, Ph.D., College of International Studies, Southwest University. 


\section{Literal Meaning or Nonliteral Meaning}

The integrational and combinational physical conditions of meaning are not only our thought, emotion, but also the act structures and image schema. The former is the congenital condition, the latter the acquired condition. Meaning happens in a very complex direct and indirect interaction and communication between man and nature, man and society, man and man. Because interaction and communication can promote people to experience meaningful pattern, employ the coherent and worldwide structural process that people can understand and their bodies create (Johnson, 1987, p. 31). So meaning generally covers literal and nonliteral cognitive structure, psychological structure, thinking structure, and image schema, which means that meaning can be literal or nonliteral and whether literal meaning or nonliteral meaning is cognitive, emotional, full of thought, and image schemata. But relatively nonliteral meaning is more abstract, often stirs or emerges in the higher level of cognitive system; literal meaning is conventionalized, often performs as linguistic expressions, and exists in the lower level of cognitive system, more exactly in the conceptualizing steps in the cognitive system, because people's language ability just belongs to the part of cognitive ability.

From speakers' perspective, undoubtedly intended meaning is also one case of meaning, because intended meaning can be literal or nonliteral. You know, speakers have the ability to use linguistic expressions to convey intended meaning more or less, because intended meaning can be resided in a linguistic expression, although it likely exists beyond a linguistic expression. So linguistic expressions sometimes can directly convey intended meaning, but sometimes linguistic expressions just act as a framework, because speakers intentionally bring meaning into the out of linguistic expressions, at this moment, such linguistic expressions are just a meaning-trigger. Meaning-trigger is like something that acts as an abstract linking force to trigger or arouse meaning from the out of linguistic expressions; it can open and achieve a meaning-resonance area shared by both speakers and listeners. Listeners can utilize the meaning-resonance area to indirectly understand speaker's intended meaning. Most cognitive science researches do not involve this field. Johnson (1987, p. 10) finds out that a lot of cognitive science works about language processing deal with the symbolization related background knowledge, but not background knowledge. In fact, background knowledge can bring people to understand linguistic expressions possibly, because background knowledge is the resources of meaning-resonance area; meaning-resonance area can be triggered and linked by the meaning-trigger acted with linguistic expressions. So linguistic meaning does not equal with intended meaning, even though linguistic expressions have the ability to convey intended meaning. Because linguistic meaning is better to take as an ideal meaning or incomplete meaning, it needs the extra meaning from the out of linguistic expressions to add or supply for our understanding; intended meaning can be seen as the sum of extra meaning and linguistic meaning.

\section{The Emergence of Meaning}

Johnson (1987, p. 5) believes that the meaning of a natural language begins in figurative and multivalent patterns; the patterns and their connection are embodied, so the meaning of a natural language cannot be simply reduced to a set of literal concepts and proposition. Because meaning includes not only embodied experience patterns, but also perceptual preconception dimension. Preconception dimension of meaning possesses public-share feature. As you know, people experience the shared dimension in the world, for example, everybody develops one certain notion, he/she should realizes everything that their bodies involve, including not only the dynamic law, orientation, emotion, but also the response of the interest, the impulse of depression, 
cultural attitude, the influence of myth, language structure, personal history, etc.

Clearly, meanings are not literal in nature; meaning possesses embodied non-propositional dimension. Experience that people' bodies create is full of not only emotion, thought, and image schemata, but also act structures created by the bodies. As an organism, in order to survive, people must influence or interact with the environment; no matter people act on objects, or objects act on people, all the interaction process is a force. So people try their best to understand experience and force structures. Force structures are similar to gravitational force, which can dominate and allocate all things and relations on the earth. Experience keeps forceful connection with each other relying on the acts created by people and other organisms. Meaning network is connected by these act structures (Johnson, 1987, p. 42), so we can say that meaning is integrated and combined by forces; meaning emerges from image schema, emotion, thought, and instant structures.

\section{Meaning From Image Schema}

According to Johnson (1987, p. 7), meaning partly comes from the shared metaphor projection. Metaphor structures can provide innate connection for conversation, partly from the multi-aspect culture "popular" mode, partly from the schema structure widely developed in the non-propositional embodied experience. These schema structures are not only the background condition for understanding, but also they are the part of meaning. Meaning is often beyond concepts and propositions. To understand intended meaning is not just reached through concepts and propositions. Because meaning comes from people' bodily experience and ordering figurative process, especially from pre-concept meaningful structure functioning, schema pattern functioning, and meaningful organization obtained from experience, as well as connecting figurative projection functioning. Through these ways, people can understand and infer meaning (Johnson, 1987, p. 17).

Here meaning in this section mainly includes schema gestalt structures. These structures are alterative flowing patterns that obtained changes from different contexts, and these patterns are abstract connecting schema that our bodies obtained in different experiences; various schema structures that formed by experience and figurative (non-proposition) program can provide coherent structures, endow meaning experience, and bless people inference (Johnson, 1987, p. 12). Typical experience patterns can cause cognitive orientation and orientate conceptualization system when these experience patterns promote to people's meaning systematic network; these patterns can provide orientated integration and abstract connecting function for linguistic expressions or interaction structures. Image schema gestalt structure can provide methods for constraint or restrict meaning, provide deduction mode for inference. Some image schema structures can be located in our meaning network through conventionalization in order to acquire certain relative stability. Based on these reasons, a great part of meaning structures can be seen as "fixed" in most times, and conventionalized meaning is usually called as "literal" meaning. But all literal meanings never escape context, because literal meaning exists based on the background including sharing schema, abilities, practice, and knowledge.

\section{Meaning From Emotion}

Meaning includes emotion structure, or meaning can emerge from emotion; such meaning is also integrated and combined by force. Force can engender innate connection in nature, suppose order and relation in advance. People depend on some relations to receive meaningful information from certain thing or matter, but relation is not the total contents of meaning. Meaning is like one point in a space; the meaning of one point can emerge from the relation between one point and another one, because the meaning of points is relevant in nature; relation among things is the meaning of these things (Ben-Ze'ev, 2001, p. 18). The relation of meaning 
implicates the comparing nature of meaning; the comparing nature of emotion meaning implicates that emotion transcend the old information, so sometimes emotion is mythological (Ben-Ze'ev, 2001, p. 35). However, emotion conveys the value and fondness of people, which means that emotion is not random (Ben-Ze'ev, 2001, 35). Logical positivists think that pre-concept dimension of meaning in force cannot ignore the meaning from emotion; emotion is bodily practical and imaginary force structure that is constituted by attitude, rhyme, mutual model, pressure notion, etc. (Johnson, 1987, p. 14). Meaning from emotion is not thoroughly separated from the connotation-denotation meaning (sense and reference; semantic-pragmatic-meaning); meaning from emotion is also not totally said as "connotation". The code of emotion in languages is the same characteristic as the encoding of connotation-denotation meaning (Wilce, 2009, p. 20). The so-called emotion language actually is not just about the description of emotion; broadly emotion experience requires people to take the same broad linguistic expression to describe these experiences. So meaning from emotion is greater than the emotion meaning of words or phrases. Meaning from emotion consists of two complementary components: the essential intention of force and the cultural contents. Cultural contents exclusively belong to culture; the force schema constructing contents are potential and ubiquitous (Kövecses, 2000, p. 189).

\section{Meaning From Thought}

Meaning must be full of thought; meaning is also the outcome resulted by thought. Because meaning can convey the notion, exhibit the wisdom, and reflect the philosophy, the world view, and the sense of worth of speakers. A linguistic expression is almost the trigger for meaning when meaning comes from the meaning-resonance area of speaker-listener; at this time, the interaction and communication are basically nonliteral in thoughts between speaker and listener. When meaning comes from the structures and semantics of linguistic expressions, the literal meaning in thoughts is often the main meaning for interaction and communication; the participant extent of nonliteral thought activities is decreased, even though the nonliteral thought activities also take part in the interaction and communication. However, the literal meaning of thought extremely limits in the sphere of interaction and communication, because the literal meaning of thought relatively commonly happens in the honest relationship among the relatives or the affiliations. Such relationship is pure, straight, and pleasant; such interpersonal interaction is free. But no matter in whatever ways, meaning is thought while meaning coming from thought takes part in interaction and communication. There is no meaning without thought.

\section{Meaning From Instant Structures}

Meaning can emerge from the instant structure that is created by activities. Instant structure can be the act, the object, the place, the way, or the state, even their composites in experience. Of course, instant structures are not only resulted by human being, but also by other organisms. Instant structure is a force; also it is an integrated and combined enabling force when people construct linguistic expressions. In a way, the meaning and the form of a linguistic expression are the correspondence of instant structures. That is, the tense and the voice of a V-structure in a linguistic expression can be projected by the act resulted by human being or other organisms; the structure and semantics of a linguistic expression can be the projection of instant structures in experience. Speakers can construct various linguistic expressions with different emphases along with different projective perspectives. When speakers are in the integration and combination process of a linguistic expression, they can directly project the visualizing meaning and the visualizing structure of instant structure to be the corresponding meaning and structure of linguistic expressions according to people's own mode of thinking and 
conventionalization. Instant structures almost keep the same in experience, because instant structures in the world can equally involve the act, the object, the place, the way, or the state, etc., although the concrete involving object, place, way, etc., are not same. So human language can be integrated and combined by instant structures with their projection, human being abides by the same rules and theories of integration and combination to construct separate human language.

All in all, meaning can come from the out of a linguistic expression or in the linguistic expression when speakers are in the interactional and communicational process, because meaning actually emerge from emotion, thought, image schema, or instant structure. Emotion, thought, image schema, and instant structure are not only the meaning emerging resources, but also the parts of meaning whether emotion, thought, image schema, and instant structure are instantiated in a linguistic expression or not. That is, the element to engender a linguistic expression is not the word, or the phrase, but the construction about emotion, thought, image schema, or instant structures.

\section{Conclusion}

Meaning can be divided as literal meaning, nonliteral meaning, linguistic meaning, or intended meaning, but actually intended meaning plays the truly interactional and communicational function. Speakers are based on intended meaning to combine and integrate their linguistic expressions when they are in the interactional and communicational process. Actually meaning emerges from emotion, thought, image schema, or instant structure. So emotion, thought, image schema, and instant structure are not only the meaning emerging resources, but also the parts of meaning of a linguistic expression. So the elementary unit of a linguistic expression is not the words, but the construction about emotion, thought, image schema, or instant structure.

\section{References}

Ben-Ze'ev, A. (2001). The subtlety of emotion. Cambridge, MA: MIT Press.

Johnson, M. (1987). The body in the mind. In The bodily basis of meaning, imagination, and reason. Chicago: The University of Chicago Press.

Kövecses, Z. (2000). Metaphor and emotion, language, culture, and body in human feeling. New York: Cambridge University Press.

Wilce, J. M. (2009). Language and thought. Cambridge: Cambridge University Press. 